\title{
Mode conversion enables optical pulling force in photonic crystal waveguides
}

Zhu, Tongtong; Novitsky, Andrey; Cao, Yongyin; Mahdy, M. R.C.; Wang, Zhong Lin ; Sun, Fangkui; Jiang, Zehui; Ding, Weiqiang

Published in:

Applied Physics Letters

Link to article, DOI:

$10.1063 / 1.4997924$

Publication date:

2017

Document Version

Publisher's PDF, also known as Version of record

Link back to DTU Orbit

Citation (APA):

Zhu, T., Novitsky, A., Cao, Y., Mahdy, M. R. C., Wang, Z. L., Sun, F., Jiang, Z., \& Ding, W. (2017). Mode conversion enables optical pulling force in photonic crystal waveguides. Applied Physics Letters, 111(6), [061105]. https://doi.org/10.1063/1.4997924

\section{General rights}

Copyright and moral rights for the publications made accessible in the public portal are retained by the authors and/or other copyright owners and it is a condition of accessing publications that users recognise and abide by the legal requirements associated with these rights.

- Users may download and print one copy of any publication from the public portal for the purpose of private study or research.

- You may not further distribute the material or use it for any profit-making activity or commercial gain

- You may freely distribute the URL identifying the publication in the public portal 


\section{Mode conversion enables optical pulling force in photonic crystal waveguides}

Tongtong Zhu, Andrey Novitsky, Yongyin Cao, M. R. C. Mahdy, Lin Wang, Fangkui Sun, Zehui Jiang, and Weiqiang Ding

Citation: Appl. Phys. Lett. 111, 061105 (2017); doi: 10.1063/1.4997924

View online: http://dx.doi.org/10.1063/1.4997924

View Table of Contents: http://aip.scitation.org/toc/apl/111/6

Published by the American Institute of Physics

\section{Articles you may be interested in}

Near-infrared linewidth narrowing in plasmonic Fano-resonant metamaterials via tuning of multipole contributions

Applied Physics Letters 111, 061104 (2017); 10.1063/1.4997423

What is the best planar cavity for maximizing coherent exciton-photon coupling

Applied Physics Letters 111, 061102 (2017); 10.1063/1.4997171

Optically active dilute-antimonide III-nitride nanostructures for optoelectronic devices

Applied Physics Letters 111, 061101 (2017); 10.1063/1.4997450

Differential ghost imaging in time domain

Applied Physics Letters 111, 061106 (2017); 10.1063/1.4997925

Cavity enhanced absorption spectroscopy in the mid-infrared using a supercontinuum source Applied Physics Letters 111, 061103 (2017); 10.1063/1.4985263

Broadband non-polarizing terahertz beam splitters with variable split ratio Applied Physics Letters 111, 071101 (2017); 10.1063/1.4986538

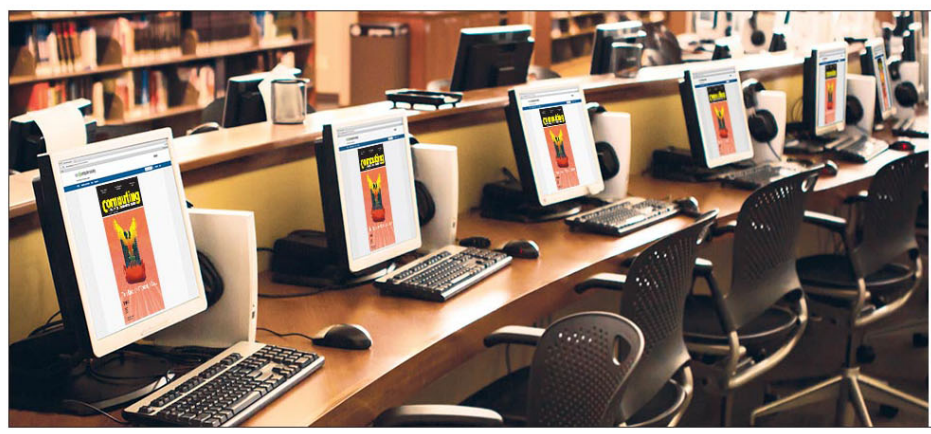

\section{CISE is already at} your fingertips...

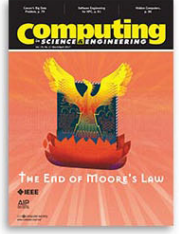

In the IEEE Xplore and

AIP library packages. 


\title{
Mode conversion enables optical pulling force in photonic crystal waveguides
}

\author{
Tongtong Zhu, ${ }^{1}$ Andrey Novitsky, ${ }^{2}$ Yongyin Cao, ${ }^{1}$ M. R. C. Mahdy, ${ }^{3,4}$ Lin Wang, ${ }^{1}$ \\ Fangkui Sun, ${ }^{1}$ Zehui Jiang, ${ }^{1}$ and Weiqiang Ding ${ }^{1, a)}$ \\ ${ }^{1}$ Department of Physics, Harbin Institute of Technology, Harbin 150001, China \\ ${ }^{2}$ DTU Fotonik, Technical University of Denmark, Orsteds Plads 343, DK-2800 Kgs. Lyngby, Denmark \\ ${ }^{3}$ Department of Electrical and Computer Engineering, North South University, Bashundhara, Dhaka 1229, \\ Bangladesh \\ ${ }^{4}$ Pi Labs Bangladesh Ltd., ARA Bhaban, 39, Kazi Nazrul Islam Avenue, Kawran Bazar, Dhaka, Bangladesh
}

(Received 22 May 2017; accepted 27 July 2017; published online 8 August 2017)

\begin{abstract}
We propose a robust scheme to achieve optical pulling force using the guiding modes supported in a hollow core double-mode photonic crystal waveguide instead of the structured optical beams in free space investigated earlier. The waveguide under consideration supports both the 0th order mode with a larger forward momentum and the 1 st order mode with a smaller forward momentum. When the 1st order mode is launched, the scattering by the object inside the waveguide results in the conversion from the 1st order mode to the 0th order mode, thus creating the optical pulling force according to the conservation of linear momentum. We present the quantitative agreement between the results derived from the mode conversion analysis and those from rigorous simulation using the finite-difference in the time-domain numerical method. Importantly, the optical pulling scheme presented here is robust and broadband with naturally occurred lateral equilibriums and has a long manipulation range. Flexibilities of the current configuration make it valuable for the optical force tailoring and optical manipulation operation, especially in microfluidic channel systems. Published by AIP Publishing. [http://dx.doi.org/10.1063/1.4997924]
\end{abstract}

Since the experimental demonstration of optical trapping and acceleration of micrometer sized objects by the optical force of laser beams, ${ }^{1,2}$ the optical manipulation has witnessed a glorious development in trapping and transporting objects ranging from the sub-nanometer scale (where the mechanical coupling of light and matter enables the cooling of atoms, ions, and molecules) ${ }^{3,4}$ to the micrometer scale (where the momentum transfer resulting from light scattering allows the trapping and manipulation of microscopic objects ${ }^{5}$ such as cells $^{6,7}$ ). Optical tweezers, for example, have been intensely exploited in multiple disciplines including atomic physics, ${ }^{8}$ biological science, ${ }^{9}$ chemistry, ${ }^{10}$ and quantum physics. ${ }^{11}$ Optical tweezers are very efficient in trapping objects around a fixed point; however, they lack flexibility for long distance transportation.

On the other hand, the counterintuitive optical pulling force, ${ }^{12-14}$ i.e., the optical scattering force with the direction reverse to the propagation of the light beam, has attracted much attention for its unique ability of optical transportation over long distances and the intriguing physics behind it. According to the conservation of linear momentum, the optical pulling force occurs only when the forward momentum of the beam is enhanced. This is the reason why the optical pulling force interacting with an ordinary passive object cannot appear for a usual plane wave or a practical Gaussian laser beam. To obtain the enhancement of the forward linear momentum and the optical pulling force, either the structured optical beams, such as a nonparaxial Bessel beam, ${ }^{12-16}$ solenoid beam, ${ }^{17}$ and interference of multiple beams, ${ }^{18-20}$ or exotic objects with optical gain, ${ }^{21,22}$ extreme anisotropy, ${ }^{21-23}$

a)wqding@hit.edu.cn chirality, ${ }^{24-27}$ and complicated metamaterial structure ${ }^{28}$ are required. Optical pulling forces are also reported in optical waveguides, where they emerge owing to the backward ${ }^{29}$ or evanescent modes, ${ }^{30-33}$ as well as coupling of the waveguide and ring resonators. ${ }^{34}$ The amplification of particle's forward momentum can be also achieved on a dielectric interface (such as airwater) ${ }^{35,36}$ and a metallic surface supporting surface plasmon polariton $^{37}$ or with the assistance of photothermal effects. ${ }^{38-41}$

In this letter, we propose a robust and distinct configuration to achieve the optical pulling force. It is grounded on the use of the guiding modes inside a multimode waveguide channel rather than the structured optical beams in free space. ${ }^{34} \mathrm{~A}$ waveguide supports eigenmodes differing in the propagation constants. For the $m$-th order mode with the propagation constant $\beta_{m}$ (the mode index $m$ is an integer), the momentum projection along the propagation direction is $\hbar \beta_{m}$ per photon and $\beta_{m}$ always decreases with the increase in $m$. When a scatterer is inserted into the waveguide, the mode conversion between different modes takes place and the optical force can be tailored by controlling the mode conversion. In particular, when the conversion occurs with the decrease in the mode index, an optical pulling force may arise according to the conservation of linear momentum. In Ref. 34, the mode conversion is also applied to achieve optical pulling force, but the object is outside the waveguide and resonantly couples with the waveguide through the evanescent field, which makes the optical pulling force inefficient and narrow band (only for the resonant frequencies). Also, the lateral equilibrium is challenging to achieve. ${ }^{34,42}$ In the current configuration of hollow core photonic crystal waveguides, all those disadvantages are suppressed. More importantly, we present the quantitative agreement between the results derived from the mode 
conversion (together with the linear momentum conservation) and the full wave numerical simulations, which depicts a clear physical picture to connect the optical pulling force and mode conversion.

It is noticed that the similar conversion of the azimuthal component of the Poynting vector to the longitudinal component was used for the explanation of the forward momentum amplification of nonparaxial Bessel beams. ${ }^{43}$ Since an infinite number of modes are excited during the scattering in this case, it is very complicated to carry out the quantitative analysis of conversion. On the contrary, with several guiding modes in the waveguide, the conversion is clearly described, allowing us to make an apparent conclusion about the pulling character of the optical force. Guided modes are beneficial from the practical point of view, too, because they are standard unlike the complicated structured optical beams and can be implemented using the well-developed techniques. The guiding structures are also efficient in energy confinement to enhance the force. ${ }^{44}$ They are also flexible and able to design a demanded particle's trajectory (e.g., a sharp bending trajectory is feasible for a photonic crystal waveguide ${ }^{45}$ ).

The hollow core photonic crystal waveguide configuration under investigation is shown in Fig. 1. The photonic crystal is formed by an array of silicon nanorods arranged in the square lattice with the lattice constant of $a$ and the radius and refractive index of the silicon nanorods being $r_{0}=0.2 a$ and $n_{a}=3.4$, respectively [see the inset in Fig. 1(a)]. The nanorods are
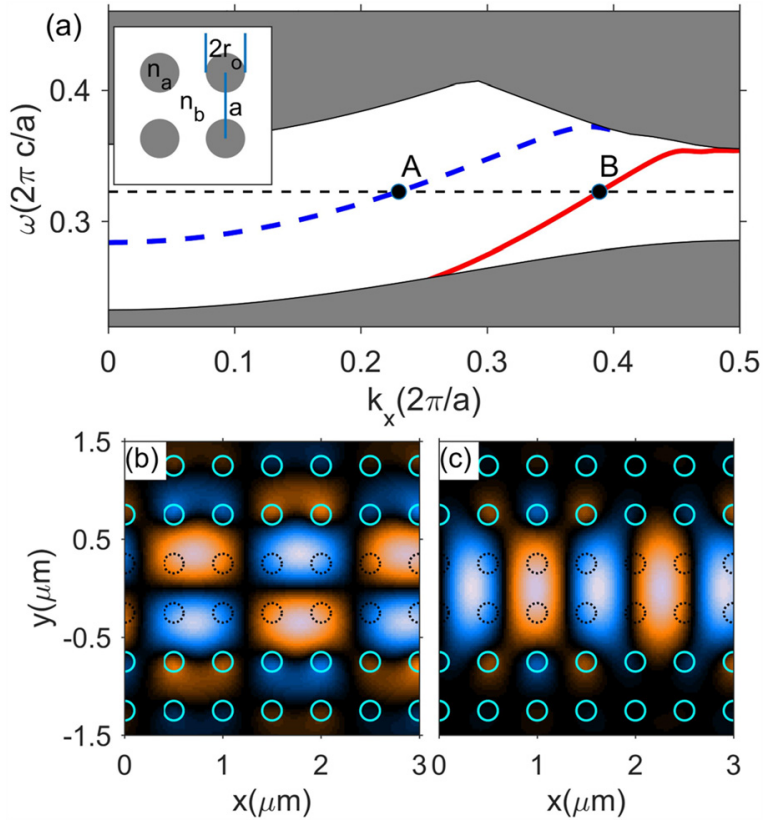

FIG. 1. (a) Band structure of TE modes in the photonic crystal (PC) waveguide. The inset shows the structure of the PC with parameters of $n_{b}=1.33$, $n_{a}=3.4$, and $r_{0}=0.2 a$ ( $a$ the lattice constant). The waveguide is fabricated by removing two lines of units along the $x$ direction, as shown in (b) and (c). At a frequency of $\omega=0.3226(2 \pi c / a)$ (dashed line), the wave vector of the 1 st (0th) order mode is $k_{x A}=0.23(2 \pi / a)\left(k_{x B}=0.39(2 \pi / a)\right)$. (b) Mode pattern of $E_{z}$ for the 1 st order mode [A in Fig. 1(a)]. The circles (cyan) show the position of the PC units, and the central two lines (gray) are removed to form the waveguide. Parameters of $a=500 \mathrm{~nm}, r_{0}=100 \mathrm{~nm}$, and $\lambda_{0}=1.5 \mu \mathrm{m}$ in vacuum [corresponding to the dashed line in (a)] are selected. Coordinate system: $y=0$ is set on the symmetry axis of the waveguide, and $x=0$ is set at $30 a$ from the front surface of the PC waveguide, where the source is launched. (c) The same as (b) but for the 0th order mode. embedded into water (refractive index, $n_{b}=1.33$ ). The photonic crystal waveguide is formed by removing two lines of rods along the $x$ direction. In Figs. 1(b) and 1(c), two central lines are removed (gray circles in color), and the nanorods of the photonic crystal are depicted with the cyan circles. We consider TE-polarized electromagnetic guided modes, of which the electric field vector has the single component $E_{z}$. The finitedifference in time-domain (FDTD) method is employed for the calculation of the band structure of TE modes. The band diagram in Fig. 1(a) clearly demonstrates the existence of the wide bandwidth, where only two guiding modes can be supported. At the normalized frequency $\omega=0.3226(2 \pi c / a)$ [marked by the horizontal dashed line in Fig. 1(a)], there are two modes denoted by the points A (1st order mode) and B (0th order mode). The wave numbers for the 0 th and 1 st order modes are about $k_{x B}=0.39(2 \pi / a)$ and $k_{x A}=0.23(2 \pi / a)$, respectively. Thus, the difference of the wave numbers $\Delta k_{x}$ of the two modes is $\Delta k_{x}=k_{x B}-k_{x A}=0.16(2 \pi / a)$. Since the forward momentum of the guiding mode is proportional to the propagation constant, the scattering induced conversion from the 1st order to Oth order will increase the forward momentum of the mode, and therefore, a backward pulling force acting on the scatterer may appear.

Setting the lattice constant $a=500 \mathrm{~nm}$ and the radius of silicon nanorods $r_{0}=100 \mathrm{~nm}$, we perform the FDTD calculation of the mode pattern $E_{z}(x, y)$ for the two modes, as shown in Figs. 1(b) and 1(c). One can observe that the structures of the modes are basically different: the 1 st order mode in Fig. 1(b) has a node on the symmetry axis of the waveguide, which is absent for the 0th order mode shown in Fig. 1(c).

The waveguide is excited using the higher order mode from the left port, which is the 1st order mode in current investigation. A two-dimensional elliptical polystyrene particle with a moderate refractive index of $n=1.55$ and semimajor axes of $r_{x}=1 \mu \mathrm{m}$ and $r_{y}=0.2 \mu \mathrm{m}$ is placed into the waveguide. When the object is located at the position of $(x, y)=(4 \mu \mathrm{m}, 0.37 \mu \mathrm{m})$, the field pattern of $\left|E_{z}\right|$ calculated using the FDTD method is shown in Fig. 2(a), which clearly demonstrates the mode conversion upon the scattering process. Since only two guiding modes are supported in this waveguide, the transition can occur only from the mode $|1\rangle$ to mode $|0\rangle$, where the Dirac notations $|0\rangle$ $=E_{z 0}(x, y) \exp \left[-i\left(\omega t-k_{x A} x\right)\right]$ (the 0th order mode) and $|1\rangle=E_{z 1}(x, y) \exp \left[-i\left(\omega t-k_{x B} x\right)\right]$ (the 1st order mode) are used for convenience. The interference pattern of the two modes is apparently observed on the right-hand side of the object in Fig. 2(a). Generally, the scattering occurs through the following four channels

$$
a|1\rangle \rightarrow b|0\rangle+c|1\rangle+d|-0\rangle+e|-1\rangle .
$$

Here, $|-1\rangle$ and $|-0\rangle$ are the modes propagating along the $-x$ direction, while $a, b, c, d$, and $e$ are the complex amplitudes of the modes. The sketch of the mode conversion process is shown in Fig. 2(b), where the levels illustrate the linear moments of the modes.

Except for the mode conversion analysis presented above, the optical force can also be accurately calculated using the integration of the stress tensor over a closed surface 

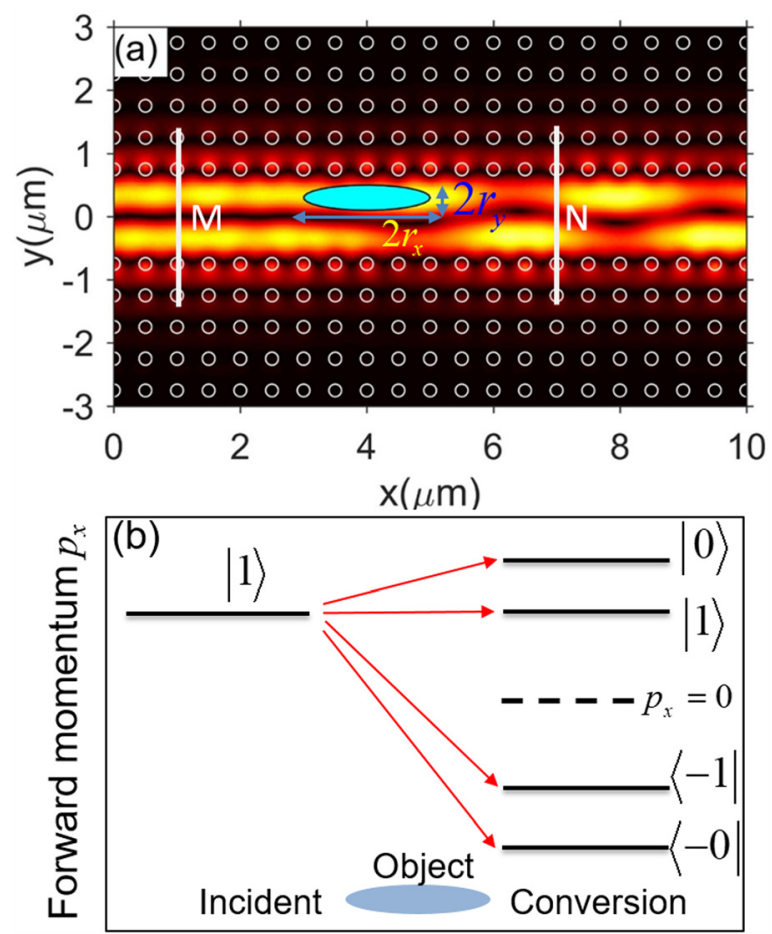

FIG. 2. (a) Field pattern $\left|E_{z}\right|$ of the 1st order mode scattered by the object. The larger ellipse is the object located at $(x, y)=(4 \mu \mathrm{m}, 0.37 \mu \mathrm{m})$, which is made of polystyrene with a moderate refractive index of $n=1.55$ and semiaxes of $r_{x}=1 \mu \mathrm{m}$ and $r_{y}=0.2 \mu \mathrm{m}$. The positions $\mathrm{M}$ and $\mathrm{N}$ are used to analyze the mode conversion and reflection. (b) Schematic of the mode conversion from the incident mode $|1\rangle$ to the other modes of $|0\rangle,|-0\rangle$, and $|-1\rangle$ (here the "minus" represents the mode to the $-x$ direction).

(a closed contour in the two dimensional case) surrounding the object, i.e.,

$$
\mathbf{F}=\oiint_{S}\langle\overleftrightarrow{\mathbf{T}}\rangle \cdot \hat{\mathbf{n}} d s
$$

where $\hat{\mathbf{n}}$ is the external unit vector normal to the surface, $\stackrel{\mathbf{T}}{\mathbf{T}}$ is the stress tensor, and $\langle\cdot\rangle$ denotes the time averaging operation over one oscillation period of the light. According to Ref. 36, the Minkowski stress tensor should be used in the current situation, which is defined as

$$
\left\langle T_{i j}\right\rangle=\frac{1}{2} \operatorname{Re}\left[D_{i} E_{j}+B_{i} H_{j}-\frac{1}{2}(\mathbf{D} \cdot \mathbf{E}+\mathbf{B} \cdot \mathbf{H}) \delta_{i j}\right],
$$

where $\delta_{i j}$ is the Kronecker delta function. Electromagnetic field strengths and inductions are obtained using the FDTD method with well tested convergence and accuracy.

Longitudinal $F_{x}$ and transverse $F_{y}$ acting on the elliptical object are shown in Fig. 3 with the parameters of $r_{a}=1 \mu \mathrm{m}$, $r_{b}=0.2 \mu \mathrm{m}$, and refractive index $n=1.55$. According to Fig. 3(a), the optical force $F_{x}$ is always negative for any positions of $x$ and $y$. The periodic oscillation of the force magnitude stems from the intrinsic nature of the periodic structure, and they share the same periodicity of $a=500 \mathrm{~nm}$, as denoted in Fig. 3(a). When the object approaches closer to the center of the waveguide $\left(y_{1}=0.15 \mu \mathrm{m}\right)$, the magnitude of the force $F_{x}$ is small. This occurs due to the fact that the 1 st order mode has a node (zero intensity) on the axis of the waveguide. For the particles farther from the waveguide center, the amplitude (absolute value) of $F_{x}$ increases (such as $y_{2}=0.2 \mu \mathrm{m}$ and
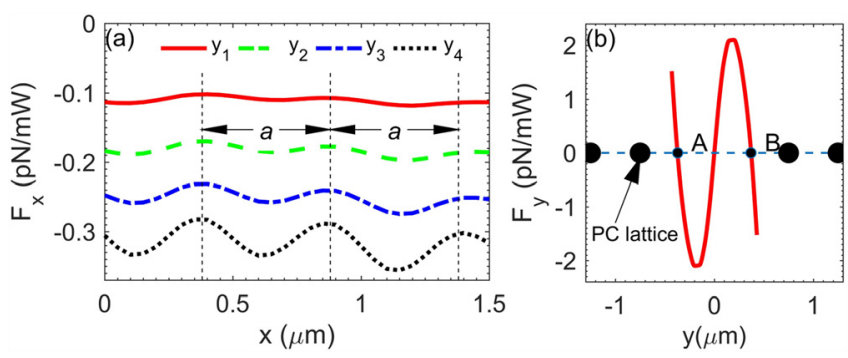

FIG. 3. Optical forces vs. the central position of the object at the fixed parameters of $r_{x}=1 \mu \mathrm{m}, r_{y}=0.2 \mu \mathrm{m}$, and $n=1.55$. (a) $F_{x}$ vs. $x$ at different transverse positions of $y_{1}=0.15 \mu \mathrm{m}$ (red solid curve), $y_{2}=0.2 \mu \mathrm{m}$ (green dashed), $y_{3}=0.25 \mu \mathrm{m}$ (blue dash-dotted), and $y_{4}=0.35 \mu \mathrm{m}$ (dark dotted). Vertical dotted lines show the periodicity of the force oscillation. (b) $F_{y}$ vs. $y$ across the waveguide at the fixed position $x=1 \mu \mathrm{m}$. $A$ and $B$ denote the two equilibrium positions of $y_{A}=-0.37 \mu \mathrm{m}$ and $y_{B}=0.37 \mu \mathrm{m}$.

$\left.y_{3}=0.25 \mu \mathrm{m}\right)$ and reaches to its maximum value around the position of $y_{4}=0.35 \mu \mathrm{m}$, where the 1 st order mode has the intensity peak.

The force $\left(F_{y}\right)$ determines the ability of the PC waveguide to trap the particle in the transverse direction. When the object moves across the waveguide at fixed $x=1 \mu \mathrm{m}$, two stable equilibrium positions marked with $\mathrm{A}$ and $\mathrm{B}$ in Fig. 3(b) can be found (the larger black dots show the position of the photonic crystal lattice). The profile of $F_{y}$ and the equilibrium positions $y= \pm 0.37 \mu \mathrm{m}$ agree well with the intensity gradient of the 1 st order mode, which indicates that the transverse force $F_{y}$ is mainly contributed by the intensity gradient force. Comparing with the configuration reported in Ref. 34, the transverse equilibrium in the current configuration occurs naturally due to the transverse restoring intensity gradient force, while the transverse equilibrium can only be achieved by using the double resonance in Ref. 42 .

It is important that the optical pulling force is broadband. As shown in Fig. 1(a), the bandwidth of the guiding modes is rather wide [from less than $0.30(2 \pi c / a)$ to $0.35(2 \pi c / a)]$ where the difference of propagation constant $\Delta k_{x}=k_{x B}-k_{x A}$ is large. This promises the optical pulling force in this whole guiding band. In order to quantitatively illustrate this property, we calculate the optical force for different wavelengths. In Fig. 4(a), the optical forces for three different wavelengths of $\lambda_{1}=1.55 \mu \mathrm{m}, \lambda_{2}=1.50 \mu \mathrm{m}$, and $\lambda_{3}=1.45 \mu \mathrm{m}$ are shown. One can see that optical pulling forces occur in this broad band with periodic modulation (with a periodicity of $500 \mathrm{~nm}$ ) in force amplitude. The broadband pulling force is a valuable feature for the practical usage.

From the point of view of momentum conservation, the reflection of the modes kicks the object in the forward direction and plays a harmful role for pulling. At the same time, the transition from $|1\rangle$ to $|0\rangle$ increases the field forward momentum and contributes to pulling. Hence, one should suppress $R$ and enhance $Q$ in order to get a larger optical pulling force. We optimize these parameters by tuning the shape of the object. Figure 4(b) shows the changes in $F_{x}$ when $r_{y}$ $=200 \mathrm{~nm}$ is fixed, while $r_{x}$ changes through $r_{x 1}=100 \mathrm{~nm}$ (red solid), $r_{x 2}=600 \mathrm{~nm}$ (green dashed), $r_{x 3}=1000 \mathrm{~nm}$ (dark dotted) to $r_{x 4}=1400 \mathrm{~nm}$ (blue dash-dotted). Except for the case of $r_{x 1}=100 \mathrm{~nm}$ (due to the small conversion efficiency and large reflections, as discussed in Fig. 5), the optical forces are 

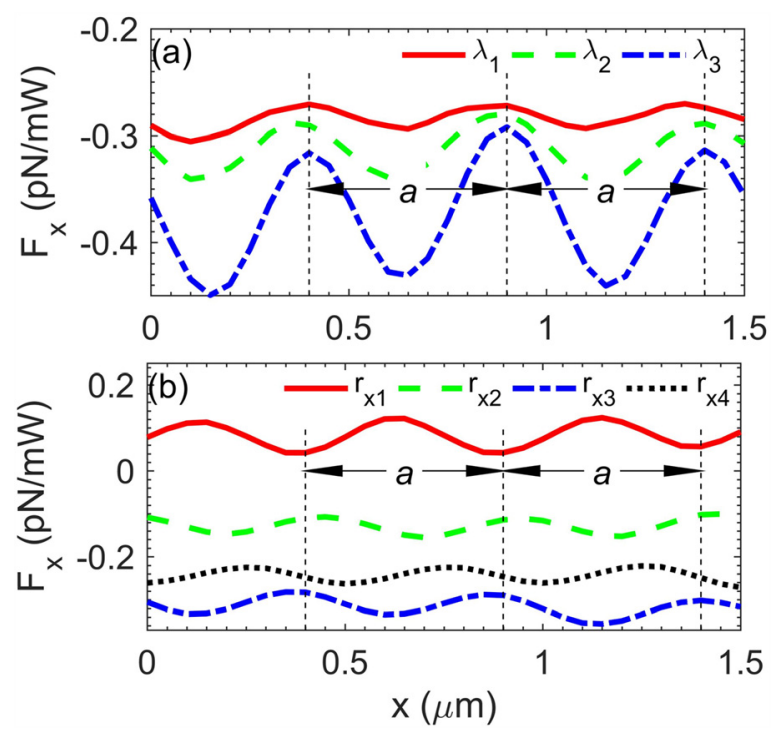

FIG. 4. (a) Broadband operation of the optical pulling force for different wavelengths of $\lambda_{1}=1.55 \mu \mathrm{m}$ (red solid curve), $\lambda_{2}=1.50 \mu \mathrm{m}$ (green dashed), and $\lambda_{3}=1.45 \mu \mathrm{m}$ (blue dash-dotted). The other parameters are the same as those used in Fig. 3, i.e., $r_{x}=1 \mu \mathrm{m}, r_{y}=0.2 \mu \mathrm{m}, n=1.55$, and $y=0.37 \mu \mathrm{m}$. (b) Changes of optical force $F_{x}$ with $r_{y}=200 \mathrm{~nm}$ and $r_{x 1}=$ $100 \mathrm{~nm}$ (red solid), $r_{x 2}=600 \mathrm{~nm}$ (green dashed), $r_{x 3}=1.0 \mu \mathrm{m}$ (blue dashdotted), and $r_{x 4}=1.4 \mu \mathrm{m}$ (dark dotted). The vertical lines show the oscillation periodicity of the force curves, which is the same as that of the photonic crystal lattice of $a$.

pulling in a wide range of $r_{x}$. When $r_{x} \sim 1000 \mathrm{~nm}$, the pulling force reaches its maximum amplitude, which is the case discussed in Figs. 3 and 4(a).

According to the mode conversion analysis presented above, one can find that the mode reflection and the conversion factors together determine the amplitude of the optical force. In order to quantitatively compare the results of mode
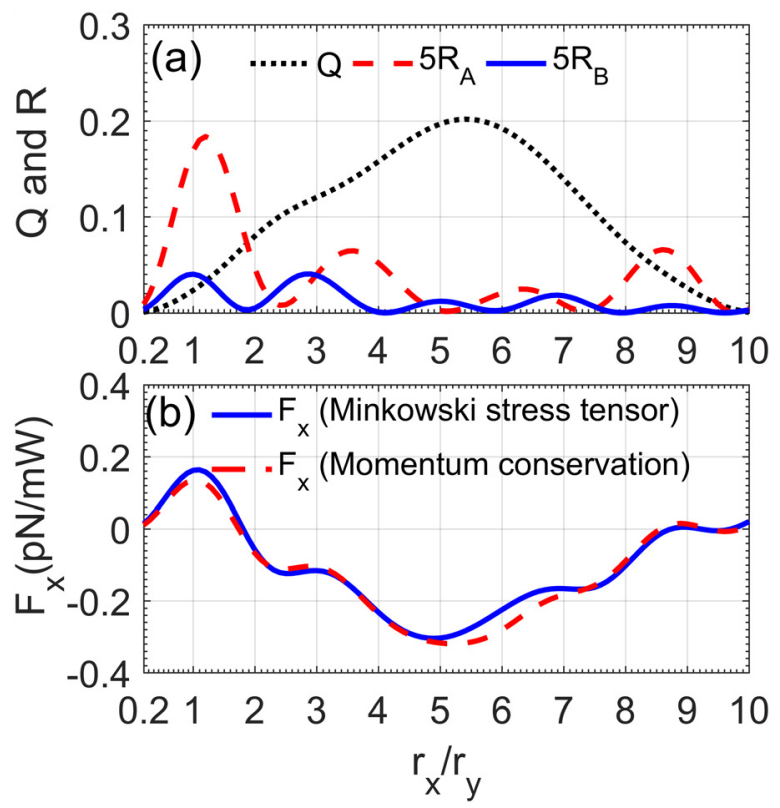

FIG. 5. (a) Mode conversion coefficients of $Q, R_{A}$, and $R_{B}$ [defined in Eq. (4)] as functions of object shapes of $r_{x} / r_{y}$ with $r_{y}=200 \mathrm{~nm}$. (b) Optical force $F_{x}$ calculated by Minkowski stress tensor (blue solid) and the optical force based on momentum conservation defined in Eq. (5) (red dashed) change with the shape parameter $r_{x} / r_{y}$ of the object. Other parameters are the same with those used in Fig. 4. conversion with those from numerical simulations, one should extract the mode reflection and conversion efficiencies accurately. For this purpose, two arbitrary positions of $M$ and $N$ to the left and right of the scatterer are selected [see Fig. 2(a)]. Then, the modes at these two positions can be expressed as

$$
|M\rangle=a|1\rangle+d|-1\rangle+e|-0\rangle \quad \text { and } \quad|N\rangle=b|0\rangle+c|1\rangle .
$$

Using the orthogonality of the modes of $\langle 1 \mid 0\rangle=\langle 0 \mid 1\rangle=0$ and $\langle 1 \mid 1\rangle=\langle 0 \mid 0\rangle=1$, the complex amplitudes of $a \sim e$ can be calculated with the assistance of the numerical simulation. Then, the mode conversion coefficient $Q$ and the reflections of $R_{A}$ (mode $A$ ) and $R_{B}$ (for mode $\mathrm{B}$ ) can be defined as

$$
Q=|b|^{2}|a|^{-2}, \quad R_{A}=|d|^{2}|a|^{-2}, \quad R_{B}=|e|^{2}|a|^{-2} .
$$

According to the conservation of linear momentum, when $N$ photons are scattered, the momentum change obtained by the object is $\Delta p_{x, \mathrm{obj}}=N \cdot \hbar\left[-\Delta k_{x} Q+2 k_{x A} R_{A}+\left(k_{x A}+k_{x B}\right) R_{B}\right]$, which is the opposite value of the momentum change of the modes. Here, $\hbar$ is the reduced Planck constant, and $\Delta k_{x}$ $=k_{x B}-k_{x A}$ with $k_{x A}\left(k_{x B}\right)$ the wave vector of the 1st (0th) mode (see Fig. 1). For the incident power of $P$, the photon number $N$ incident on the object in a time period of $\Delta t$ is $N=P$ $\cdot \Delta t /(\hbar \omega)$ with $\omega$ the angular frequency. Therefore, the optical force $F_{x}$ experienced by the object is

$$
F_{x}=\frac{\Delta p_{x, \mathrm{obj}}}{\Delta t}=\frac{P}{\omega}\left[-\Delta k_{x} Q+2 k_{x A} R_{A}+\left(k_{x A}+k_{x B}\right) R_{B}\right] .
$$

Here, $Q, R_{A}$, and $R_{B}$ are defined in Eq. (4).

Figure 5(a) shows the changes of $Q$ and $R_{A}$ and $R_{B}$ in the case of $r_{y}=0.2 \mu \mathrm{m}$ and $r_{x}=\xi r_{y}$. From Fig. 5(a), one can observe that the mode conversion reaches the maximum value around $\xi=5$, i.e., $r_{x}=1.0 \mu \mathrm{m}$. When those parameters are substituted into Eq. (5), the optical force we defined based on momentum conservation is calculated and shown in Fig. 5(b) (red dashed). For comparison, the force $F_{x}$ calculated using the numerical simulation of the FDTD method (Minkowski stress tensor) is also shown in Fig. 5(b) (blue solid). Quantitative agreement between them is obtained. For smaller $\xi$ (such as $\xi<1.8$ ), the mode conversion $Q$ is insufficient to overcome the effect of the mode reflection. Thus, we end up with the optical pushing force. In the case of $\xi \approx 5$, maximum $F_{x}$ is achieved since the mode conversion $Q$ is the largest while mode reflections $R_{A}$ and $R_{B}$ are small, and this is just the case used in Figs. 3 and 4.

In summary, we have proposed a distinct configuration, which is a two dimensional hollow core photonic crystal waveguide supporting both the 0th and 1st order modes, to achieve optical pulling force by using the mode conversion. Since the lower order mode carries more forward linear momentum than the higher order modes, the optical pulling force is achieved by suppressing the mode reflection and enhancing the mode conversion from the 1 st to the 0th mode. We have calculated the optical pulling force according to the mode conversion and linear momentum conservation, and quantitative agreement with the results of the finitedifference in the time-domain method is obtained, which constructed a clear and accurate physical picture to connect the optical pulling force and the mode conversion. 
Importantly, the current configuration is broadband, robust to the object shape, and high efficient in pulling force generation. Also, the lateral equilibriums can occur naturally due to the lateral restoring intensity gradient force. This work provides an advantageous way for the flexible tailoring of optical pulling force, which may find potential applications, especially in microfluidic channels and related domains.

This work was supported by the National Natural Science Foundation of China (NSFC) (11474077 and 11404083) and Program for Innovation Research of Science in Harbin Institute of Technology (A201411 and B201407). A.N. acknowledges partial financial support from the Villum Fonden (project DarkSILD).

${ }^{1}$ A. Ashkin, J. M. Dziedzic, J. E. Bjorkholm, and S. Chu, Opt. Lett. 11(5), 288 (1986).

${ }^{2}$ A. Ashkin, Phys. Rev. Lett. 24(4), 156-159 (1970).

${ }^{3}$ S. Stellmer, B. Pasquiou, R. Grimm, and F. Schreck, Phys. Rev. Lett. 110(26), 263003 (2013).

${ }^{4}$ D. G. Grier, Nature 424(6950), 810-816 (2003).

${ }^{5}$ H. Xin, R. Xu, and B. Li, Sci. Rep. 2,818 (2012).

${ }^{6}$ J. Guck, R. Ananthakrishnan, T. J. Moon, C. C. Cunningham, and J. Kas, Phys. Rev. Lett. 84(23), 5451-5454 (2000).

${ }^{7}$ M. C. Zhong, X. B. Wei, J. H. Zhou, Z. Q. Wang, and Y. M. Li, Nat. Commun. 4, 1768 (2013)

${ }^{8}$ S. Chu, Science 253(5022), 861-866 (1991).

${ }^{9}$ O. M. Marago, P. H. Jones, P. G. Gucciardi, G. Volpe, and A. C. Ferrari, Nat. Nanotechnol. 8(11), 807-819 (2013).

${ }^{10}$ K. C. Neuman and A. Nagy, Nat. Methods 5(6), 491-505 (2008).

${ }^{11}$ J. Chan, T. P. Alegre, A. H. Safavi-Naeini, J. T. Hill, A. Krause, S. Groblacher, M. Aspelmeyer, and O. Painter, Nature 478(7367), 89-92 (2011).

${ }^{12}$ J. Chen, J. Ng, Z. Lin, and C. T. Chan, Nat. Photonics 5, 531-534 (2011).

${ }^{13}$ A. Dogariu, S. Sukhov, and J. J. Sáenz, Nat. Photonics 7, 24-27 (2013).

${ }^{14}$ A. Novitsky, C.-W. Qiu, and H. Wang, Phys. Rev. Lett. 107(20), 203601 (2011).

${ }^{15}$ S. Sukhov and A. Dogariu, Opt. Lett. 35, 3847-3849 (2010).

${ }^{16}$ N. Wang, J. Chen, S. Liu, and Z. Lin, Phys. Rev. A 87(6), 063812 (2013).

${ }^{17}$ S.-H. Lee, Y. Roichman, and D. G. Grier, Opt. Express 18(7), 6988-6993 (2010).
${ }^{18}$ O. Brzobohatý, V. Karásek, M. Šiler, L. Chvátal, T. Čižmár, and P. Zemánek, Nat. Photonics 7(2), 123-127 (2013).

${ }^{19}$ S. Sukhov and A. Dogariu, Phys. Rev. Lett. 107(20), 203602 (2011).

${ }^{20}$ D. B. Ruffner and D. G. Grier, Phys. Rev. Lett. 109(16), 163903 (2012).

${ }^{21}$ K. J. Webb and Shivanand, Phys. Rev. E 84(5), 057602 (2011).

${ }^{22}$ A. Mizrahi and Y. Fainman, Opt. Lett. 35(20), 3405-3407 (2010).

${ }^{23}$ A. Novitsky and C.-W. Qiu, Phys. Rev. A 90(5), 053815 (2014).

${ }^{24}$ K. Ding, J. Ng, L. Zhou, and C. T. Chan, Phys. Rev. A 89(6), 063825 (2014).

${ }^{25}$ A. Canaguier-Durand and C. Genet, Phys. Rev. A 92(4), 043823 (2015).

${ }^{26} \mathrm{G}$. Li, M. Wang, H. Li, M. Yu, Y. Dong, and J. Xu, Opt. Mater. Express 6(2), 388 (2016).

${ }^{27}$ M. Wang, H. Li, D. Gao, L. Gao, J. Xu, and C. Qiu, Opt. Express 23(13), 16546-16553 (2015).

${ }^{28}$ A. S. Shalin, S. V. Sukhov, A. A. Bogdanov, P. A. Belov, and P. Ginzburg, Phys. Rev. A 91(6), 063830 (2015).

${ }^{29}$ A. V. Maslov, Phys. Rev. Lett. 112(11), 113903 (2014).

${ }^{30}$ N. K. Paul and B. A. Kemp, Prog. Electromagn. Res. 151, 73-81 (2015).

${ }^{31}$ T. Zhu, M. R. Mahdy, Y. Cao, H. Lv, F. Sun, Z. Jiang, and W. Ding, Opt. Express 24(16), 18436-18444 (2016).

${ }^{32}$ N. K. Paul and B. A. Kemp, Opt. Eng. 55(1), 015106 (2016).

${ }^{33}$ T. Zhang, S. Mei, Q. Wang, H. Liu, C. T. Lim, and J. Teng, Nanoscale 9(20), 6895-6900 (2017).

${ }^{34}$ V. Intaraprasonk and S. Fan, Opt. Lett. 38(17), 3264-3267 (2013).

${ }^{35}$ V. Kajorndejnukul, W. Ding, S. Sukhov, C.-W. Qiu, and A. Dogariu, Nat. Photonics 7(10), 787-790 (2013).

${ }^{36}$ C.-W. Qiu, W. Ding, M. R. C. Mahdy, D. Gao, T. Zhang, F. C. Cheong, A. Dogariu, Z. Wang, and C. T. Lim, Light: Sci. Appl. 4(4), e278 (2015).

${ }^{37}$ M. I. Petrov, S. V. Sukhov, A. A. Bogdanov, A. S. Shalin, and A. Dogariu, Laser Photonics Rev. 10(1), 116-122 (2016).

${ }^{38}$ C. Wang, Z. Gong, Y.-L. Pan, and G. Videen, Appl. Phys. Lett. 109(1), 011905 (2016).

${ }^{39}$ V. Shvedov, A. R. Davoyan, C. Hnatovsky, N. Engheta, and W. Krolikowski, Nat. Photonics 8(11), 846-850 (2014).

${ }^{40}$ J. Lu, H. Yang, L. Zhou, Y. Yang, S. Luo, Q. Li, and M. Qiu, Phys. Rev. Lett. 118(4), 043601 (2017).

${ }^{41}$ O. Ilic, I. Kaminer, Y. Lahini, H. Buljan, and M. Soljačić, ACS Photonics 3(2), 197-202 (2016).

${ }^{42}$ V. Intaraprasonk and S. Fan, Opt. Express 21(21), 25257-25270 (2013).

${ }^{43}$ D. Gao, A. Novitsky, T. Zhang, F. C. Cheong, L. Gao, C. T. Lim, B. Luk'yanchuk, and C.-W. Qiu, Laser Photonics Rev. 9(1), 75-82 (2015).

${ }^{44}$ M. G. Scullion, Y. Arita, T. F. Krauss, and K. Dholakia, Optica 2(9), 816 (2015).

${ }^{45}$ A. Mekis, J. C. Chen, I. Kurland, S. Fan, P. R. Villeneuve, and J. D. Joannopoulos, Phys. Rev. Lett. 77(18), 3787-3790 (1996). 